\begin{tabular}{|c|l|}
\hline Title & UItrasensitive detection of organophosphate insecticides by carbon nanotube field-effect transistor \\
\hline Author(s) & Ishii, A tsushi; Takeda, Seiji; Hattori, Satoshi; Sueoka, Kazuhisa; Mukasa, Koichi \\
\hline Citation & $\begin{array}{l}\text { Colloids and Surfaces A Physicochemical and Engineering A spects, 313, 456-460 } \\
\text { https://doi.org/L0.1016/.colsurfa.2007.05.071 }\end{array}$ \\
\hline Issue Date & 2007-02-01 \\
\hline Doc URL & http://hdl.handle.net/2115/32365 \\
\hline Type & article (author version) \\
\hline File Information & cs_ishii_etal.pdf \\
\hline
\end{tabular}

Instructions for use 


\title{
Ultrasensitive detection of organophosphate insecticides by carbon nanotube field-effect transistor
}

\author{
Atsushi Ishii*a ${ }^{\text {, Seiji Takeda }}{ }^{\mathrm{a}}$, Satoshi Hattori ${ }^{\mathrm{a}}$, Kazuhisa Sueoka $^{\mathrm{b}}$, Koichi Mukasa ${ }^{\mathrm{a}}$ \\ ${ }^{a}$ Creative Research Initiative “Sousei”, Hokkaido University, Sapporo 001-0021, Japan \\ ${ }^{b}$ Graduate School of Information Science and Technology, Hokkaido University, \\ Sapporo 060-0814, Japan
}

PACS: 85.30.Tv; 87.15.Rn; 87.480.-y

Keywords: Field effect transistor; Biochemistry; Biosensors

\footnotetext{
* Corresponding author. Tel. +81-11-706-9295; fax: +81-11-706-9294.

E-mail address: ishii@cris.hokudai.ac.jp (A. Ishii)
} 


\begin{abstract}
Carbon nanotube sensors were capable of detecting organophosphate insecticide binding to recombinant acetylcholinesterase (rAChE) immobilized on the sensor. The carbon nanotube sensors were fabricated by chemical vapor deposition method and it showed field effect transistor property. rAChE was immobilized by specific binding of NTA-Ni and His tag on the reverse side of the carbon nanotube sensor. The current between the source and drain was measured after incubation of various concentrations of organophosphates with immobilized rAChE. $I-V_{\text {gate }}$ curves were obtained by plotting the current as a function of the potential applied to the back gate. The $I-V_{\text {gate }}$ curves showed a positive shift in a dose dependent manner, while no shifts were observed when an herbicide was added to the sensor and organophosphates were withdrawn on recombinant calmodulin immobilized surface. In spite of different inhibition effects, two organophosphates showed similar dose response curves in measuring $I-V_{\text {gate }}$ correlation with the rAChE-immobilized CNT sensors, suggesting that the binding of organophosphates to rAChE may be sequestered from the inhibition activity of those to the enzyme.
\end{abstract}




\section{Introduction}

Insecticides are widely employed in agriculture in order to protect from the harmful insect damage of farm products. Organophosphates and carbamates, which are approximately two-thirds of insecticides used [1], act as suicide substrates for acetylcholinesterase (AChE), hence preventing the termination of a nerve impulse in the postsynaptic membrane. These insecticides are potentially toxic not only to insects but also other animals including humans. Of particular concern is the exposure of infants and children to food contaminants because of their possible increased susceptibility to adverse effects [2, 3]. It is, therefore, important to develop the sensing device that is highly sensitive for residual agricultural chemical emergency.

Coupling gas chromatography with mass spectrometry is expensive and time consuming. For rapid and low-cost detection of organophosphate and carbamate compounds, enzyme based methods has also been investigated. Several efforts involving the introduction of mutation in various AChEs by protein engineering have been made to increase the sensitivity of AChE biosensors toward many organophosphates and carbamates [4-6]. Schulze et al. reported the highly sensitive detections of important insecticides at concentration below $10 \mu \mathrm{g} / \mathrm{kg}$, using biosensors composed of artificial AChE in combination with the amperometric transducers [6].

Tans et al. firstly demonstrated the possibility of using a semiconducting single wall carbon nanotube (SWCNT) as a field effect transisitor (FET) [7]. The subsequent development of chemical methods to directly or indirectly immobilize recognition moieties to the electric transducer allowed the functionalization of CNT, leading to practical use as new types of sensor of CNT-FET for biomolecules. Actually, some biological materials including proteins [8], nucleic acids [9], viruses[10], etc, have been 
detected with CNT-FET based sensors. Recently, we showed that antigen-antiboddy interactions occurring on the reverse side of CNT were detectable with a CNT-FET in extraordinary high sensitivity $[11,12]$. To extend the availability of our developed CNT-FET sensors for biological use, we attempted the detection of interaction between AChE and insecticides on the reverse side of the CNT.

\section{Experiment}

\subsection{Materials}

$N$-[5-(3'-Maleimidopropylamido)-1-carboxypentyl]iminodiacetic acid, disodium salt, and monohydrate (Maleimide NTA) were purchased from Dojindo Laboratories, Japan. 3-Mercaptopropyltriethoxysilane (S810) was obtained from Chisso Corp., Japan. Other materials were purchased from Sigma-Aldrich Co., Japan.

\subsection{Fabrication of CNT-FET device}

The CNT-FET device was fabricated by a combination of position-controlled growth of CNTs, photo-lithography, and electron beam deposition. Formation of the chemical catalyst, CNT growth, and deposition of metallic electrode was carried out according to the methods described previously [11].

\subsection{Construction of Expression Vectors for His-Tagged AChE}

Drosophila melanogaster poly(A) ${ }^{+} \mathrm{RNA}$ was reverse-transcribed with random hexamers. To produce an truncated form of AChE, PCR was carried out using the first

strand

cDNAs

as

template,

and

as

primers,

5’-GGGGCGGCCGCATGGCCATCTCCT-GTCGGCAGAGCAGAG-3’

nucleotides 1,089 to 1,116 containing a Not I site (underlined) ) and 5’-ATGGTCGACGAGCCTCGGGGATATGGAAGCACTTCCC-3’' (complementary to 
nucleotides 2,945 to 2,972 containing a Sal I site (underlined)). The PCR product was digested with Not I and Sal I, followed by subcloning into Not I- Sal I site of pBAD/gIII-C (Invitrogen). pFastBac ${ }^{\mathrm{TM}} 1$ (Invitrogen) was digested with $\mathrm{Xba}$ I, filled in, and further digested with Not I. The DNA fragment encoding the partial $D$. melanogaster AChE with His tag at the C-terminus was subcloned into pFastBac ${ }^{\mathrm{TM}} 1$. Preparation of a recombinant bacmid DNA containing the partial D. melanogaster AChE sequence, transfection of Sf9 insect cells with the bacmid DNA, and secretion of the His-tagged AChE to serum-free medium (Express Five ${ }^{\mathrm{TM}}$ ) from High Five cells were carried out according to the manufacture's protocol.

\subsection{Preparation of rAChE and recombinant Calmodulin (rCaM)}

E. coli TOP10 was transformed with $\mathrm{pBAD} / \mathrm{gIII} / \mathrm{calmodulin}$ (Invitrogen). 0.02\% L-arabinose was added to the culture of the bacteria to induce an expression of the His-tagged CaM. The rCaM and the rAChE were purified by affinity chromatography on Ni-NTA column (Qiagen). In the former case, further purification was performed by gel filtration with Superdex ${ }^{\mathrm{TM}} 75$ and AKTA system (GE Healthcare Bioscience).

\subsection{Modification of the reverse side of the CNT}

The modification of the reverse side of the CNT with NTA-Ni was carried out according to the methods described previously [11]. Fifty $\mu \mathrm{l}$ of $7 \mu \mathrm{g} / \mathrm{ml}$ recombinant protein(s) was dropped on the surface, and incubated for $10 \mathrm{~min}$ at room temperature. After washing with PBS, $50 \mu$ l of various concentrations of agricultural chemicals were dropped onto the surface, and incubated for $10 \mathrm{~min}$ at room temperature.

\subsection{Measurements}

In order to examine the properties of CNT, the current between the source and drain $\left(I_{\mathrm{sd}}\right)$ was measured in air by applying $-20 \mathrm{~V}$ to $20 \mathrm{~V}$ to the back gate that faced the 
reverse side of the CNTs. The schematic model of the $I-V_{\text {gate }}$ measurements is shown in Fig. 1. Organophosphates or herbicide samples were prepared by stepwise dilution of each $10 \mathrm{mg} / \mathrm{L}$ organophosphate or herbicide in acetone with PBS. The $I_{\mathrm{sd}}$ was plotted as a function of the potential applied to the back gate ( $I-V_{\text {gate }}$ curve). Various concentrations of agricultural chemicals were withdrawn on the recombinant proteins-immobilized surface of the CNT-FET sensor and incubated for $10 \mathrm{~min}$. After washing the surface with PBS and drying up, an Agilent 4155C semiconductor characterization system was employed to measure the $I-V_{\text {gate }}$ curves at room temperature.

\section{Results and discussion}

We investigated the properties of the CNT grown on the substrate by measuring the $I$ - $V_{\text {gate }}$ curves while changing the potential of the reverse side of the CNT. The $I_{\mathrm{sd}}$ was dependent on the potential of the back gate, as in a FET device. The FET property of the CNT sensor is consistent with the characteristics reported previously [11]. The CNT showed conductive or semi-conductive properties depending on the CNT chirality. In this experiment, $I_{\mathrm{sd}}$ increased when the back gate potential decreased to $-20 \mathrm{~V}$, suggesting that $I_{\mathrm{sd}}$ was strongly affected by the effective potential around the CNT.

The $I-V_{\text {gate }}$ curves were measured by the addition of serial dilutions of two organophosphates (acephate and fenitrothion) to the rAChE-immobilized surface (Fig. $2 \mathrm{a}$ and $2 \mathrm{~b}$ ). The error of measurement of each reaction processes was estimated to be less than $0.5 \mu \mathrm{A}$, as deduced from the $I-V_{\text {gate }}$ curve noise. The error of the shift of the $I$ - $V_{\text {gate }}$ curves of the same CNT sensor is less than $1 \mathrm{~V}$. Properties of different CNT sensors are dissimilar, as are their sensitivities. These phenomena might be attributable 
to contact between the CNT and metal electrode, besides the chirality of the CNT.

Both in acephate and fenitrothion, $I-V_{\text {gate }}$ curves showed a positive shift after the addition of organophosphate solutions in the concentration range of $1 \mathrm{pg} / \mathrm{L}$ to $1 \mathrm{ng} / \mathrm{L}$. This suggests that the effective potential around CNT may change to positive due to the production of an enzyme-inhibitor complex between the organophosphates and rAChE. Fig 2c shows the $I-V_{\text {gate }}$ curves after loading of an herbicide (pretilchlor) on the CNT sensor as a control. In this case, only nonspecific interaction of the herbicide to the sensor surface was observed. As shown in Fig. 2c, the $I-V_{\text {gate }}$ curves did not directionally shift after the addition of pretilchlor and so there was no clear correlation between the shift of the $I-V_{\text {gate }}$ curves and the concentration of organophosphates as indicated in Fig. 2a and 2b. To confirm the specific interaction between organophosphates and the rAChE, acephate and fenitrothion solutions were dropped on the rCaM-immobilized surface, which is unrelated protein with organophosphates, followed by measuring $I-V_{\text {gate }}$ profiles. As shown in Fig. 3a and $3 \mathrm{~b}, I-V_{\text {gate }}$ curves shifted slightly without directional change. This suggests that nonspecific binding to the surface appeared to be negligible and that the shifts of the $I-V_{\text {gate }}$ curves depicted in Fig. $2 \mathrm{a}$ and $2 \mathrm{~b}$ were due to specific interaction of organophosphates to the immobilized rAChE, which may have changed the effective potential around the CNT to positive.

The $I_{\text {sd }}$ is plotted as a function of agricultural chemicals concentrations to quantify the $I-V_{\text {gate }}$ curve shifts at $0 \mathrm{~V}$ (Fig. $4 \mathrm{a}, 4 \mathrm{c}$ to $4 \mathrm{e}$ ) or at $4.77 \mathrm{~V}$ (Fig. $4 \mathrm{~b}$ ). Figure 4 clearly shows that the $I-V_{\text {gate }}$ curves shifted in the positive direction in the range from $1 \mathrm{pg} / \mathrm{L}$ to $1 \mathrm{ng} / \mathrm{L}$ and in the negative direction in the range from $1 \mathrm{ng} / \mathrm{L}$ to $100 \mathrm{ng} / \mathrm{L}$. Control experiments (Fig. 4c, 4d and 4e)) suggested that $I_{\text {sd }}$ ranged from 0.27 to $0.92 \mu \mathrm{A}$ as results of measurement error and nonspecific binding of organophosphates to the 
substrate surface.

To evaluate the inhibition of the rAChE by the organophosphates used, enzyme activity was assayed spectrophotometricaly [14]. After incubation of the rAChE with the same concentrations of organophosphates tested in the CNT-FET senor for $10 \mathrm{~min}$ at room temperature, the remaining activity was determined (Table I). The rAChE was more than 100-fold susceptible to fenitrothion than to acephate. On the other hand, the herbicide showed no reduced activity to the rAChE. The sensitivity of the CNT sensors for detection of organophosphates was 10,000-fold higher than that of optical method. In spite of different inhibition effects, acephate and fenitrothion showed similar dose response curves in measuring $I-V_{\text {gate }}$ correlation with the rAChE-immobilized CNT sensors, suggesting that the binding of organophosphates to rAChE may be sequestered from the inhibition activity of those to the enzyme. Three-dimensional structure analysis of AChE revealed that insecticide binds serine located at catalytic triad where occupy the bottom of the gorge. It has, not yet, been elucidated the stoichiometry of binding between organophosphates and AChE except that at the active site. The interaction of organophosphates to the immobilized rAChE may have occurred at multiple sites accompanying without any inhibitory effect. Investigation of the binding mechanism of organophosphates and AChE, and subsequent conformational change of AChE should be performed in future studies.

The $I-V_{\text {gate }}$ curves shifted in the negative direction in high organophosphate concentrations are problem for AChE sensor. This will also be a future task. One means to avoid an erroneous decision might be preparation of a few diluted samples. Organophosphate concentrations in a high concentration range or low concentration range will be determined by measuring two or three diluted samples. 
The lines in Fig. 4a and 4b are the tentative calibration curves of the CNT sensor. The detection limit of acephate and fenitrothion using the CNT sensor was estimated to be an approximately $10 \mathrm{pg} / \mathrm{L}$ from the tentative calibration line, which is more than 100-fold sensitive compared with the biosensors composed of rAChE in combination with the amperometric transducers [7].

\section{Conclusion}

Ultrasensitive detection of organophosphate insecticides is possible with a combination of the CNT sensor developed by us and AChE without any site-directed mutagenesis.

\section{Acknowledgments}

This work was supported by the Grant-in-Aid for Exploratory Research on Agriculture Areas No. 17658081 from the Ministry of Education, Science and Culture, Japan, and by the Special Coordination Funds for Promoting Science and Technology from the Cabinet Office, Government of Japan. 


\section{References}

[1] D. Donaldson, T. Kieloy, A. Grube, Pesticides: 1998-1999 Pesticide Market Estimates; U. S. Environmental Protection Agency: Washington, DC, 2002.

[2] S. Padilla, J. Buzzard, V. C. Moser, Neurotoxicology 21, (2000) 49.

[3] S. Padilla, H. J. Sung, V. C. Moser, Toxicol. Environ. Health, Part A 67, (2004) 1477.

[4] T. T. Bachmann, R. D. Schmid, Anal. Chim. Acta 401, (1999) 95.

[5] Y. Boublik, P. Saint-Aguet, A. Lougarre, M. Arnaud, F Villatte, S. Estrada-Mondaca, D. Fournier, Protein Eng. 15, (2002) 43.

[6] H. Schulze, S. B. Muench, F. Villatte, R. D. Schmid, T. T. Bachmann, Anal. Chem. 77, (2005) 5823.

[7] S. J. Tans, A. R. M. Vershueren, C. Dekker, Nature 393, (1998) 49.

[8] A. Star, J-C. P. Gabriel, K. Bradley, G. Gruner, Nano Lett. 3 (2003) 459.

[9] M. Zheng, A. Jagota, M. S. Strano, A. P. Santos, P. Barone, S. G. Chou, B. A. Diner, M. S. Dresselhaus, R. S. Mclean, G. B. Onoa, G. G. Samsonidze, E. D. Semke, M. Usrey, D. J. Walls, Science 302, (2003) 1545.

[10] G. Gruner, Anal. Bioanal. Chem. 384, (2006) 322.

[11] S. Takeda, A. Sbagyo, Y. Sakoda, A. Ishii, M. Sawamura, K. Sueoka, H. Kida, K. Mukasa, K. Matsumoto, Biosens. Bioelectron. 21, (2005) 201.

[12] S. Takeda, H. Ozaki, S. Hattori, A. Ishii, H. Kida, K. Mukasa, J. Nanosci. Nanotechno. (2006) 752.

[13] NCBI sequence data bank, accession No. NM_057605

[14] G. L. Ellmann, K. D. Courtney, V. Andres, R. M. Featherstone, Biochem. Pharmacol. 7, (1961) 88. 


\section{Figure Captions}

Fig. 1. Schematic model of $I-V_{\text {gate }}$ measurements using the CNT sensors. The $I-V_{\text {gate }}$ were measured in air and a voltage from $-20 \mathrm{~V}$ to $20 \mathrm{~V}$ was applied to the back gate.

Fig. 2. $I-V_{\text {gate }}$ profiles on the binding of organophosphates to rAChE immobilized on the sensors. Acephate (a) and fenitrothion (b) was added to rAChE immobilized surface of the CNT sensor. As a control, pretilchlor was dropped on the similar sensor (c).

Fig. 3. $I-V_{\text {gate }}$ profiles on the binding of organophosphates to $\mathrm{d}$ rCaM immobilize on the sensors. Acephate (a) and fenitrothion (b) was added to rCaM immobilized surface of the CNT sensor.

Fig. 4. Dose-dependency of $I_{\mathrm{sd}}$ on the detection of organophosphates with the $\mathrm{C}$ NT sensors. Current $\left(I_{\mathrm{sd}}\right)$ of the $I-V_{\text {gate }}$ curves was plotted at $0 \mathrm{~V}$ (a, c, d, and e) or at $4.77 \mathrm{~V}$ (b). Acephate (a, d) and fenitrothion (b, e) was added to rAChE (a, b) or to rCaM immobilized surface of the CNT sensors. Pretilchlor was also added to rAChE immobilized surface of the sensor. 
Table 1. Inhibition of AChE by organophosphates

The remaining activity was determined after incubation of $\mathrm{rAChE}$ with variou s concentrations of organophosphates and a herbicide for 10 min at room te mperature.

\begin{tabular}{rccc}
\hline \multirow{2}{*}{ Inhibitor Conc } & \multicolumn{3}{c}{ AChE Activity $\left(\times 10^{6} \boldsymbol{\mu m o l} / \mathbf{m l} / \mathbf{m i n}\right)$} \\
\cline { 2 - 4 } & Acephate & Fenitrothion & Pretilchlor \\
\hline 10 ppq & 1.50 & 1.38 & 1.13 \\
100 ppq & 1.47 & 1.35 & 1.17 \\
1 ppt & 1.51 & 1.37 & 1.16 \\
10 ppt & 1.53 & 1.37 & 1.10 \\
100 ppt & 1.55 & 1.18 & 1.19 \\
1 ppb & 1.49 & 0.49 & 1.12 \\
10 ppb & 1.41 & 0.07 & 1.09 \\
\hline
\end{tabular}

Table 1. Ishii, et al 


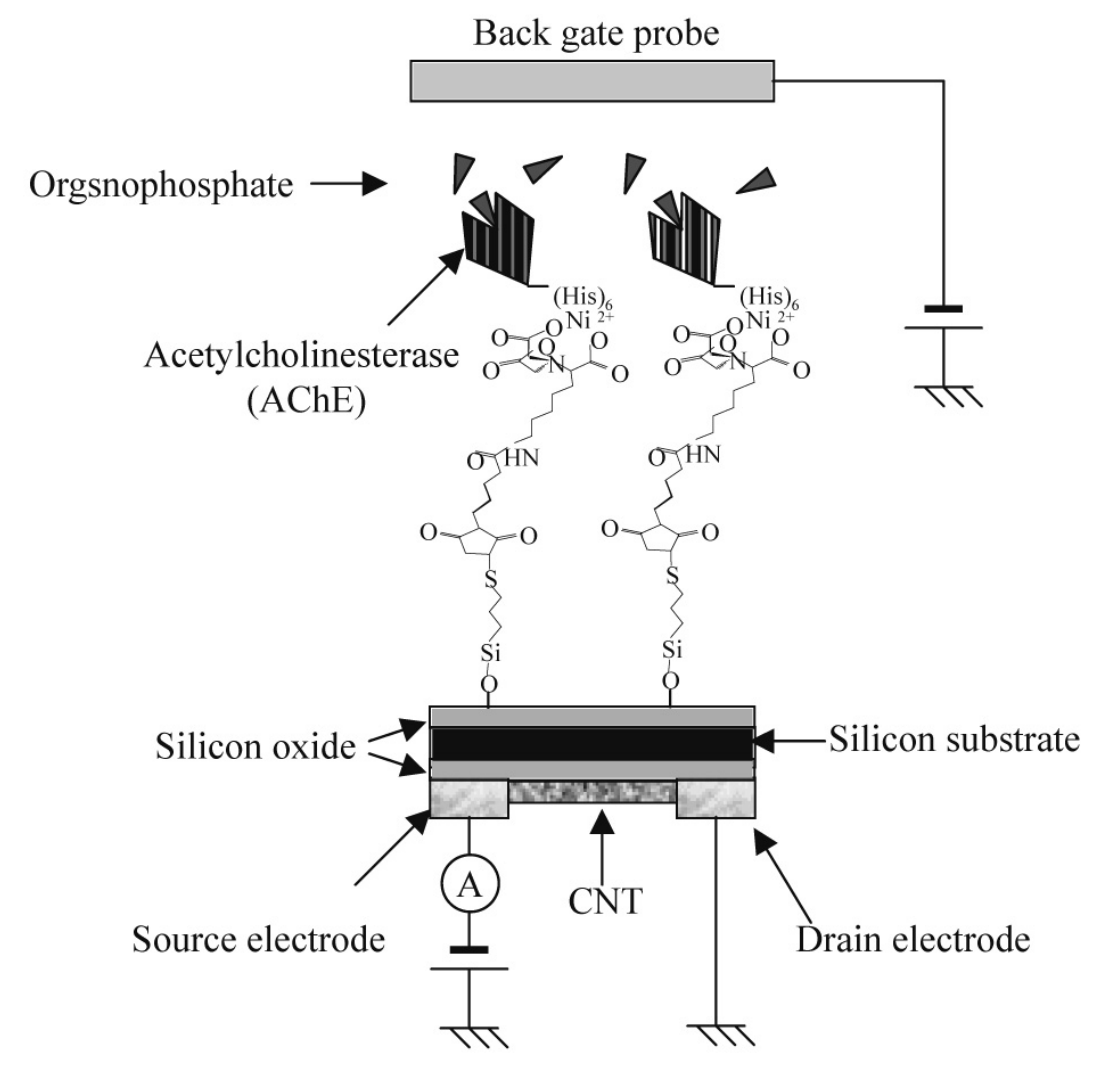

Fig. 1. Ishii, et al 

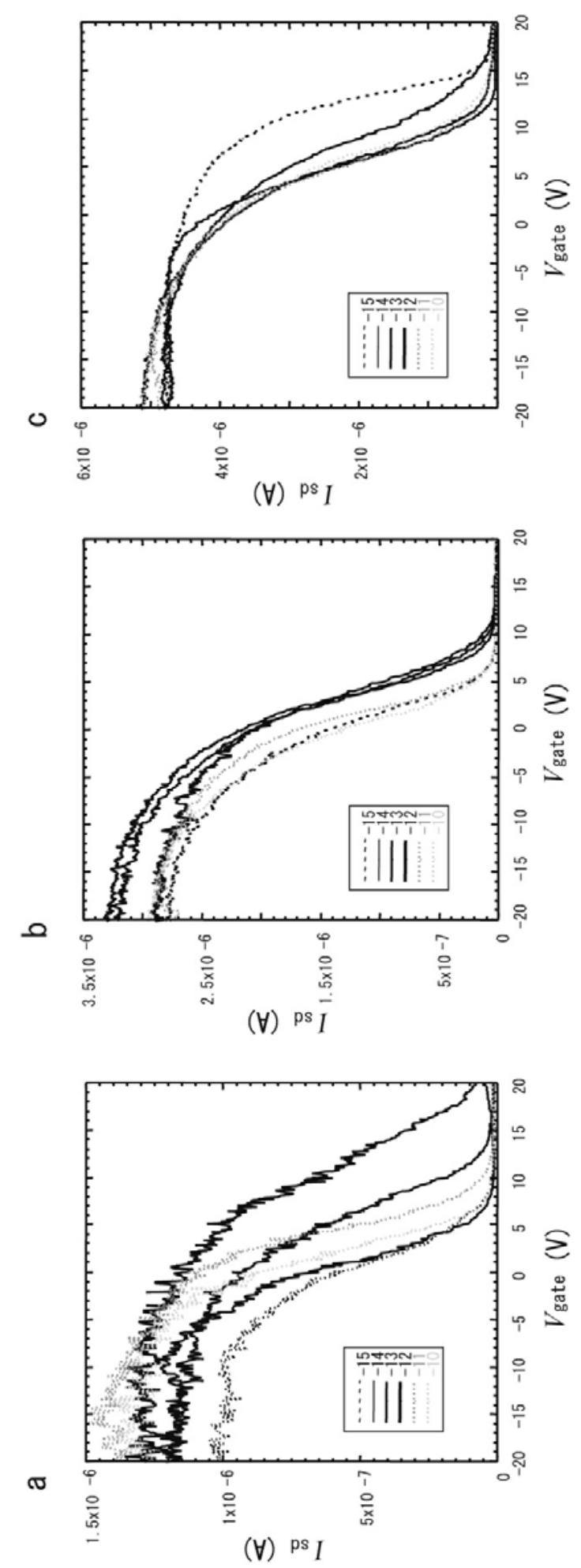

Fig. 2. Ishii, et al 

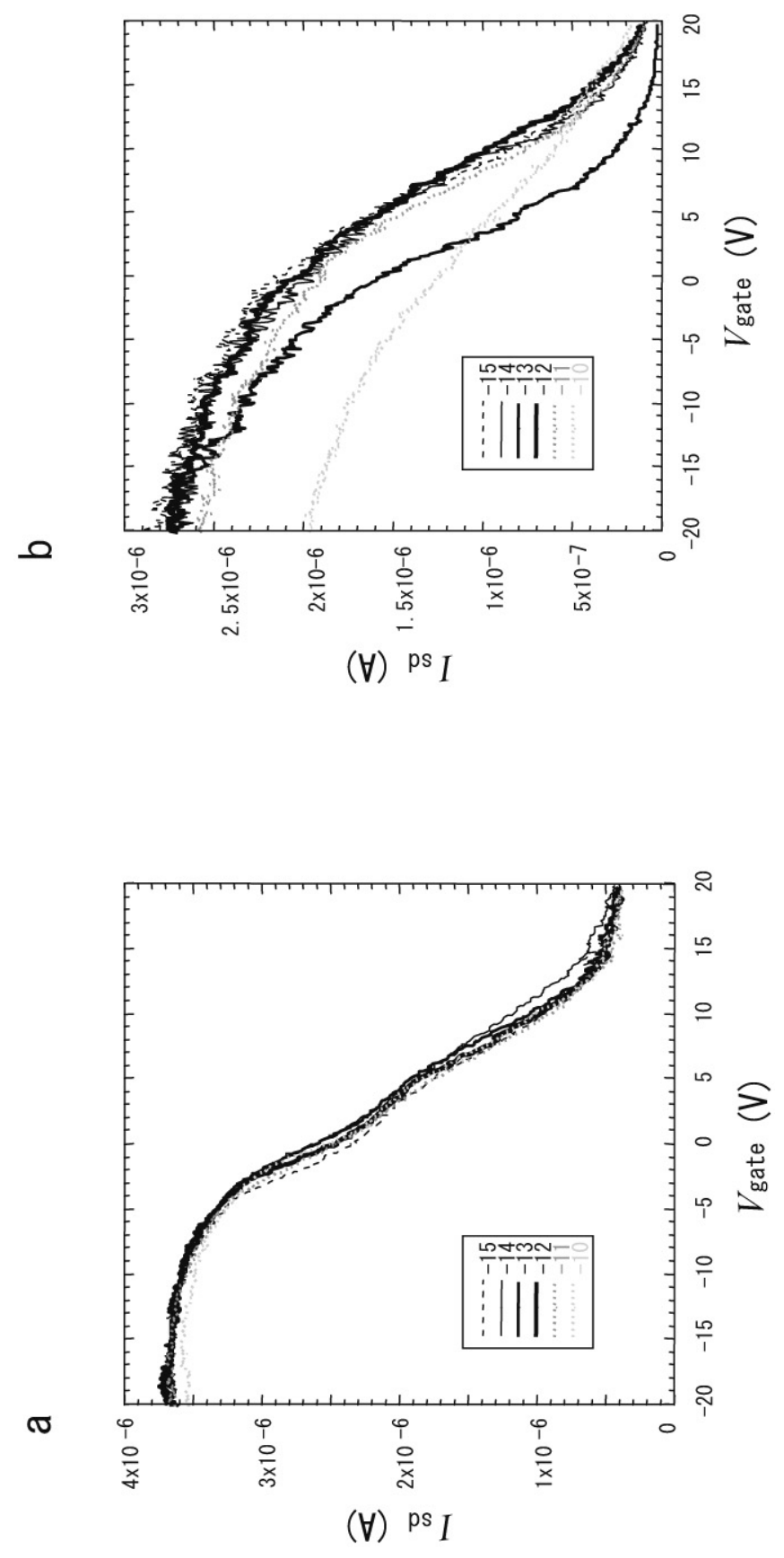

Fig. 3. Ishii, et al 


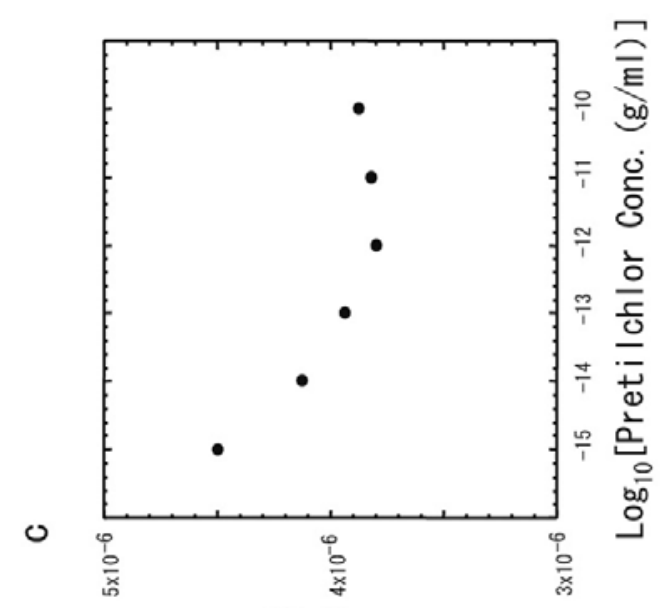

(V) ${ }^{\mathrm{ps}} I$
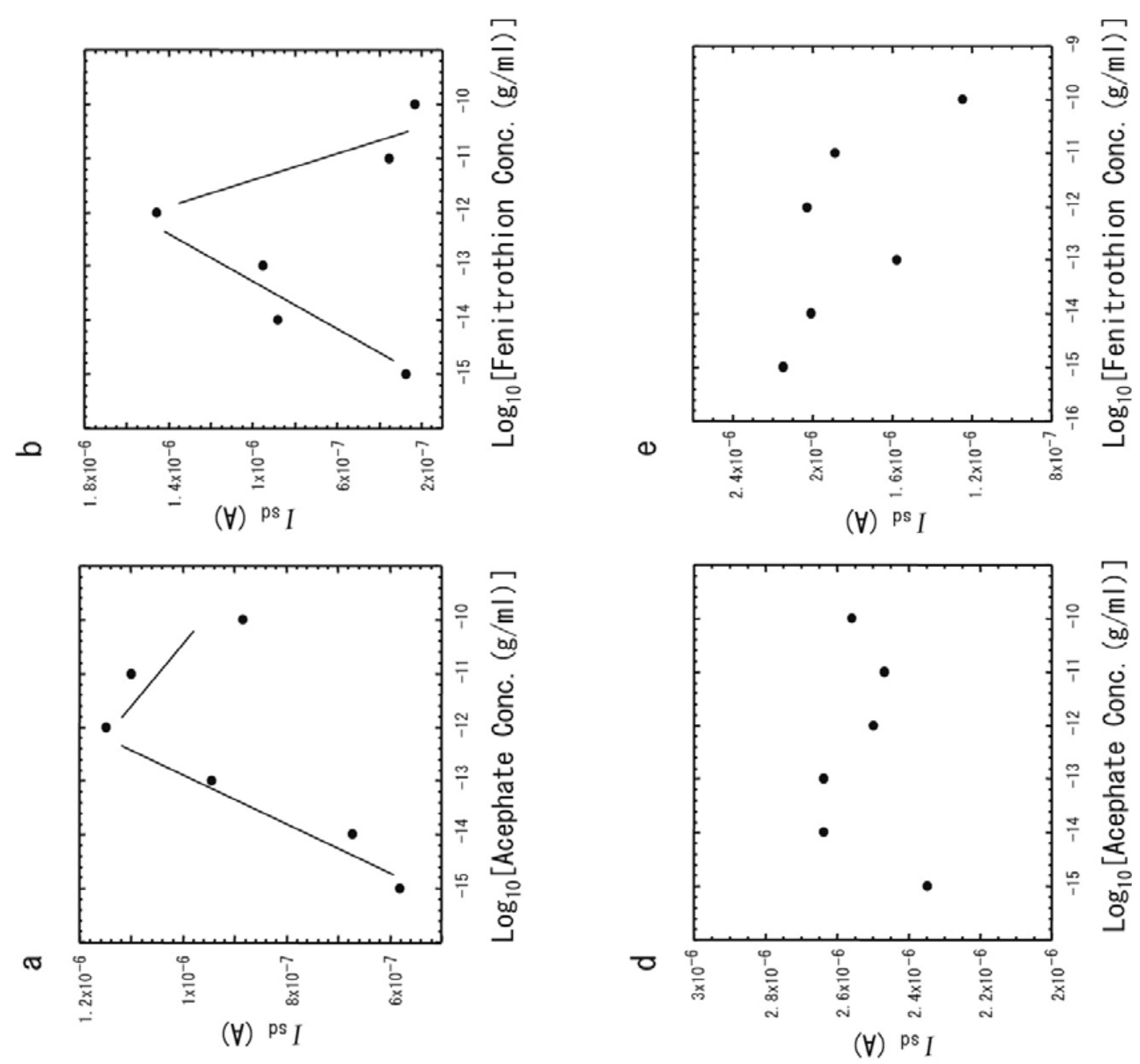

Fig. 4. Ishii, et al 